\title{
EEE Project - Students from all parts of peninsula collaborate to study cosmic rays
}

\author{
S.Grazzi $i^{1, *}$, M.Abbrescia ${ }^{1,2}$, C.Avanzini $i^{1 ; 3}$, L.Baldini $i^{1,3}$, R.Baldini Ferroli $i^{1,4}$, \\ G.Batignani $i^{1,3}$, M.Battaglieri ${ }^{1,17}$, S.Boi ${ }^{1,8}$, E.Bossini $i^{1,5}$, F.Carnesecchi ${ }^{1,6}$, \\ A.Chiavassa ${ }^{1,7}$, C.Cicalo ${ }^{1,8}$, L. Cifarelli ${ }^{1,6}$, F.Coccetti ${ }^{1}$, E.Coccia ${ }^{1,9}$, A.Corvaglia ${ }^{1,10}$, \\ D.De Gruttola1 ${ }^{1}$, S. De Pasquale ${ }^{1,11}$, F.L.Fabbri ${ }^{1,4}$, V.Frolov ${ }^{16}$, P.Galeotti ${ }^{1,7}$, \\ M.Garbini ${ }^{1,6}$, G.Gemme ${ }^{1,17}$, I.Gnesi ${ }^{1,7}$, C.Gustavino ${ }^{1,12}$, D.Hatzifotiadou ${ }^{1,6,15}$, P.La \\ Rocca $^{1,18}$, G.Mandaglio ${ }^{1,19}$, O.Maragoto Rodriguez ${ }^{14}$, G.Maron ${ }^{13}$, M.N.Mazziotta ${ }^{1,20}$, \\ S.Miozzi ${ }^{1,4}$, R.Nania ${ }^{1,6}$, F.Noferini ${ }^{1,6}$, F.Nozzoli ${ }^{1,21}$, F.Palmonari ${ }^{1,6}$, M.Panareo ${ }^{1,10}$, \\ M.P.Panetta ${ }^{1,10}$, R. Paoletti ${ }^{1,5}$, W.Park ${ }^{14}$, L.Perasso ${ }^{1,17}$, F.Pilo ${ }^{1,3}$, G.Piragino ${ }^{1,7}$, \\ S.Pisano ${ }^{1,4}$, F.Riggi ${ }^{1,18}$, G.C.Righini ${ }^{1}$, C.Ripoli ${ }^{1,11}$, M.Rizzi ${ }^{1 ; 2}$, G.Sartorelli ${ }^{1,6}$, \\ E.Scapparone ${ }^{1,6}$, M.Schioppa ${ }^{1,22}$, A.Scribano ${ }^{1,3}$, M.Selvi ${ }^{1,6}$, S.Serci $^{1,8}$, S.Squarcia ${ }^{1,17}$, \\ M.Taiuti ${ }^{1,17}$, G.Terreni ${ }^{1,3}$, A.Trifirò ${ }^{1,23}$, M.Trimarchi ${ }^{1,23}$, M.C.Vistoli ${ }^{13}$, L.Votano ${ }^{1,12}$, \\ M.C.S.Williams ${ }^{1,6,15}$, L.Zheng ${ }^{1,14,15}$, A.Zichichi ${ }^{1,6,15}$, R.Zuyeuski ${ }^{1,15}$
}

(1) Museo Storico della Fisica e Centro Studi e Ricerche Enrico Fermi, Roma, Italy

(2) INFN and Dipartimento Interateneo di Fisica, Università di Bari, Bari, Italy

(3) INFN and Dipartimento di Fisica, Università di Pisa, Pisa, Italy

(4) INFN, Laboratori Nazionali di Frascati, Frascati (RM), Italy

(5) INFN Gruppo Collegato di Siena and Dipartimento di Fisica, Università di Siena, Siena, Italy

(6) INFN and Dipartimento di Fisica e Astronomia, Università di Bologna, Bologna, Italy

(7) INFN and Dipartimento di Fisica, Università di Torino, Torino, Italy

(8) INFN and Dipartimento di Fisica, Università di Cagliari, Cagliari, Italy

(9) INFN and Dipartimento di Fisica, Università di Roma Tor Vergata, Roma, Italy

(10) INFN and Dipartimento di Matematica e Fisica, Università del Salento, Lecce, Italy

(11) INFN and Dipartimento di Fisica, Università di Salerno, Salerno, Italy

(12) INFN, Laboratori Nazionali del Gran Sasso, Assergi (AQ), Italy

(13) INFN CNAF, Bologna, Italy

(14) ICSC World Laboratory, Geneva, Switzerland

(15) CERN, Geneva, Switzerland

(16) JINR Joint Institute for Nuclear Research, Dubna, Russia

(17) INFN and Dipartimento di Fisica, Università di Genova, Genova, Italy

(18) INFN and Dipartimento di Fisica e Astronomia, Università di Catania, Catania, Italy

(19) INFN Sezione di Catania and Dipartimento di Scienze Chimiche, Biologiche, Farmaceutiche e Ambientali, Università di Messina, Messina, Italy

(20) INFN Sezione di Bari, Bari, Italy

(21) INFN and ASI Science Data Center, Roma, Italy

(22) INFN and Dipartimento di Fisica, Università della Calabria, Cosenza, Italy

(23) INFN Sezione di Catania and Dipartimento di Scienze Matematiche e Informatiche, Scienze Fisiche e Scienze della Terra, Università di Messina, Messina, Italy

The European Physical Society Conference on High Energy Physics

5-12 July, 2017

Venice

*Speaker : Stefano Grazzi, stefano.grazzi@ge.infn.it

(c) Copyright owned by the author(s) under the terms of the Creative Commons

Attribution-NonCommercial-NoDerivatives 4.0 International License (CC BY-NC-ND 4.0). 


\section{Introduction}

The Project Extreme Energy Events - Science inside Schools (EEE) [1][2] is an experiment dedicated to the study of the high energy cosmic radiation, using muon detectors distributed over an area of more than half a million $\mathrm{km}^{2}$, from Geneva to Lampedusa. Data are taken simultaneously from about 50 stations. In each station, 3 MRPCs detectors [3] positioned vertically one above the others, at 50 $\mathrm{cm}$ distance, and its electronics, constitute the "Telescope" of the EEE project. More than 50 billion particles have been registered up to now. As a physics experiment, several analyses have been performed $\&$ published:

- Search coincidences between near telescopes [4]

- Study muon flux decrease due solar events [5][6]

- Study of Anisotropy at sub-TeV scale [7]

- Study of muon decay into up-going events [8]



Figure 1 - Distribution of institutes with telescope (in red), without (in blue) and INFN sections with detectors.

- Search for long distance correlations (hundred km)

- Determination of telescopes performance [9]

The Project was born in 2004 from an idea of Professor Antonino Zichichi, funded and operated by the Museo Storico della Fisica e Centro Studi e Ricerche 'Enrico Fermi' [1] in Rome, in collaboration with CERN, INFN, and the Ministry of Education, with the aim also to involve high school students in a high energy experiment in operation. The Initiative involves more than 100 participating educational institutes, with a support of 10-20 students per school. Half of these schools already hosts a detector, while others are involved in data taking and analysis together with twinned schools.

\section{Detector Construction and Monitoring}

The participation of students starts with the construction of the detectors at CERN, working with local researchers. The construction takes five days during which each team build the three MRPC detectors needed for their telescope. During this phase, they learn how it is possible to build a particle detector using common materials such as glass, plastic and fishing lines. After construction, the detectors are brought in Italy and installed inside

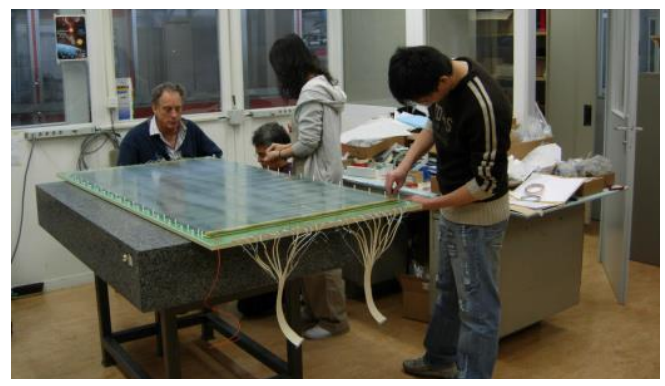

Figure 2- Photo with students, teachers and researchers during the construction of a telescope at CERN

their school. With the active telescope, students perform day-to-day checks on various aspects of the telescope, such as: Data acquisition, Hits distribution, Environment parameters, High and Low Voltages values, Gas System. 
This daily work is very important, particularly during periods of coordinated data taking (RUN, usually from October to June), when as much good data as possible with the greatest number of operational stations are being collected. Student work was a key factor at this stage and allowed us to reach $100 \mathrm{M}$ tracks per day.

\section{Calibration \& active measures}

Student activities are not limited to monitoring work but also include practical activities and measurements. Some of these are also needed to evaluate the behavior of the telescope and to set some working parameters. It is important, for example, to measure the orientation of the detector relative to the geographic north to correct muons arrival direction data. It is also necessary, one or twice per year, to measure the telescope efficiency, which is used in official analysis and also allows us to determine the optimal voltage to be provided to the detector. Students are also free to take advantage of the project to carry out their own experimental activities. In some cases the institutes, in particular without a telescope, use small scintillator detectors (called Cosmic Box) for activities such as measurement

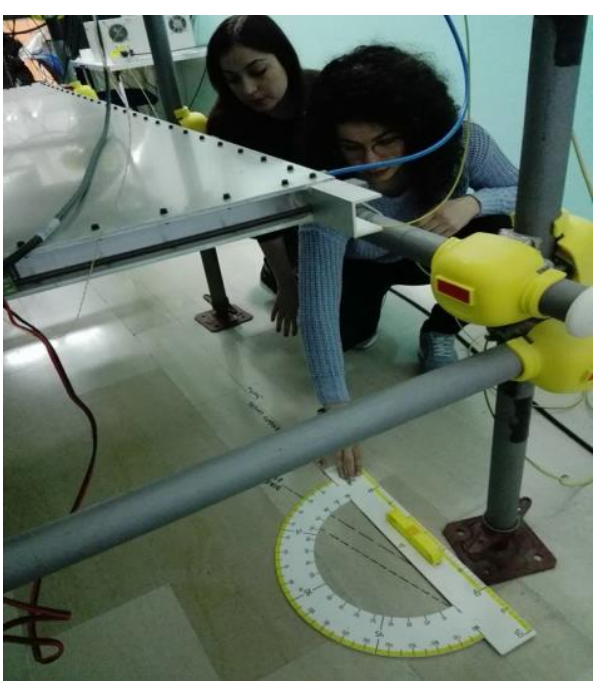

Figure 3 - Photo of students measuring the telescope's orientation of the telescope acceptance and cosmic ray flux variation with altitude, detector inclination \& orientation, different kind and thicknesses of overlying material ...

\section{Data analysis activity}

To better understand the work of a researcher, students carry out their own studies by analyzing telescopes data. In order to make accessible to all institutes, the information collected by the detectors, but also to improve the data quality monitoring, a web page [10] was created, where it is possible to find the data collected by all the telescopes:

- Telescope rates (trigger, good tracks)

- Tracks parameters ( $\chi^{2}$, time-of-flight, muon direction)

- Atmospheric parameters

However, it is difficult for this activity to be carried out by all institutes without proper preparation. In some cases, it is not only necessary to explain the analysis you want to do but also to teach how to use the tools necessary to this activities (in particular computational tools). To help teachers and students in the various stages of the analysis, various EEE Masterclasses have been prepared: Features of high energy cosmic showers, Hunting extreme events on Sun, Looking for telescopes coincidences, Statistics, Reference system transformation... 


\section{Meetings \& Symposia}

Finally, in order to give schools the opportunity to present their work, to confront the other institutes and to encourage collaboration between the students, several meetings are organized. In addition to regular meetings between nearby schools, there are also conferences (even videoconferencing) that involve all the schools at the same time. During these meetings, students are also informed on the status of the project and the scientific results. Once a year, all schools partecipate to a general meeting in Erice hosted by Fondazione e Centro di Cultura Scientifica "Ettore Majorana", during which they also conduct targeted exercises on various aspects of the project and the physics of cosmic rays. These activities are aimed at stimulating the interest of students, but also at

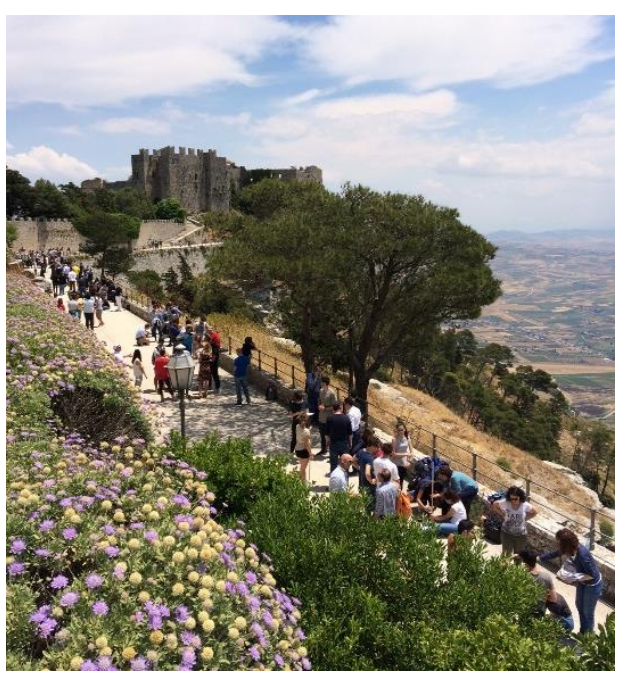

Figure 4 - Photo of the students during an exercise in last EEE General Meeting held in Erice at the end of May 2017 fostering collaboration among all the institutes.

\section{References}

[1] Centro Fermi web site: http://www.centrofermi.it/eee.

[2] A. Zichichi, Progetto "La Scienza nelle Scuole" - EEE: Extreme Energy Events, SIF (2004)

[3] M. Abbrescia et al. (EEE Collaboration) JINST (2012) 7 P11011.

[4] M. Abbrescia et al. (EEE Collaboration), Nuovo Cimento 125 (2010) 243.

[5] M. Abbrescia et al. (EEE Collaboration), Eur. Phys. J. Plus (2011) 126, 61.

[6] M. Abbrescia et al. (EEE Collaboration), Eur. Phys. J. Plus (2013) 128, 62.

[7] M. Abbrescia et al. (EEE Collaboration), Eur. Phys. J. Plus (2015) 130, 187.

[8] M. Abbrescia et al. (EEE Collaboration), Nucl. Instrum. Meth. A 816 (2016) 142-148.

[9] M. Abbrescia et al. (EEE Collaboration), Nucl. Instrum. Meth. A 845 (2017) 383.

[10] http://eee.centrofermi.it/monitor 